University of Nebraska - Lincoln

DigitalCommons@University of Nebraska - Lincoln

\title{
Searching Image in the Pigeon: A Test of Three Hypothetical Mechanisms
}

\author{
Alan B. Bond \\ University of Nebraska - Lincoln, abond1@unl.edu \\ Donald A. Riley \\ University of California, Berkeley
}

Follow this and additional works at: https://digitalcommons.unl.edu/bioscibehavior

Part of the Behavior and Ethology Commons

Bond, Alan B. and Riley, Donald A., "Searching Image in the Pigeon: A Test of Three Hypothetical Mechanisms" (1991). Papers in Behavior and Biological Sciences. 37.

https://digitalcommons.unl.edu/bioscibehavior/37

This Article is brought to you for free and open access by the Papers in the Biological Sciences at DigitalCommons@University of Nebraska - Lincoln. It has been accepted for inclusion in Papers in Behavior and Biological Sciences by an authorized administrator of DigitalCommons@University of Nebraska - Lincoln. 
Ethology 87, 203-224 (1991)

C1991 Paul Parey Scientific Publishers, Berlin and Hamburg ISSN 0179-1613

University of Nebraska State Museum, Lincoln, and

Department of Psychology, University of California, Berkeley

\title{
Searching Image in the Pigeon: A Test of Three Hypothetical Mechanisms
}

\author{
Alan B. Bond \& Donald A. Riley
}

B OND, A. B. \& RiLEY, D. A. 1991: Searching image in the pigeon: A test of three hypothetical mechanisms. Ethology 87, 203-224.

\begin{abstract}
The searching image hypothesis was originally proposed to account for the observation that animals selecting among disparate foods often consume an excess of the more common types. The hypothesis states that animals searching for a particular cryptic food item focus on visual features that are characteristic of that item, thereby facilitating its discrimination from the background. A change in stimulus discriminability is not, however, the only feasible explanation for the effect. One alternative is a simple change in response bias, an increased predisposition to respond to food-related stimuli. Another possible hypothesis derives from the fact that the amount of time that an animal has available for examining each stimulus is not fixed. This "caution" hypothesis attributes improvements in stimulus detection to chan ges in the amount of evidence that the animal acquires before making a response.

To distinguish among these alternatives, we developed an accumulator model of visual search, which included discriminability, response bias, and caution as explicit, independent components. The consequences of varying each parameter in the model were evaluated by computer simulation, generating predicted effects of each hypothetical mechanism on response time and accuracy. The predictions were then tested using pigeons searching for images of natural, cryptic food grains. The results provide strong support for the discriminability hypothesis, but suggest that secondary changes in caution may also be involved.

Corresponding author: ALAN B. Bond, University of Nebraska State Museum, W436 Nebraska Hall, UNL, Lincoln, NE 68588-0514, U.S.A.
\end{abstract}

\section{Introduction}

Animals that search for a variety of dissimilar food types often show frequencydependent selection: Foods that are relatively uncommon in the environment tend to be underrepresented in the diet, while more abundant foods are consumed in excess (KREBS 1973; MURDOCH \& OATEN 1974; CURIO 1976; GREENWOOD \& ElTON 1979). TINBERGEN (1960) proposed that this selection bias reflects the adoption of a "searching image", a perceptual change that improves the animal's ability to discriminate food-related stimuli from a background containing features of a similar appearance. He hypothesized that stimuli that were detected more often would be more readily discriminated than those with which the animal had had less experience, thereby assur- 
ing a higher frequency of selection for relatively abundant food types.

Much of the research addressed to TINBERGEN's concept has concentrated on the initial acquisition of a feeding response to novel food types (HARVEY et al. 1975; FULLICK \& GREENWOOD 1979; WILLIS et al. 1980) or on learning to discriminate a familiar food in novel, cryptic circumstances (D AWKINS 1971a; LAWRENCE 1985 a,b). It has not commonly been recognized, however, that such processes alone cannot produce a sustained frequency-dependent selection. Unless the learning were fairly volatile, assuring that the appearance of known, rare food types could easily be forgotten, the animal would eventually learn to recognize all foods in its environment and would subsequently consume them in direct proportion to their natural abundance.

Maintenance of frequency-dependent selection requires a mechanism that operates continuously, even when the animal is thoroughly familiar with all available foods, and that responds rapidly to shifts in relative abundance. A number of researchers (CROZE 1970; DAWKINS 1971b; KREBS 1973, PIETREWICZ \& KAMIL 1979; BOND 1983; BLOUGH 1989) have interpreted TINBERGEN's perceptual change as involving initial discrimination learning and subsequent shifts in selective attention among alternative food stimuli. The differences in resulting dynamics between response acquisition and attentional shifts are illustrated in PIETREWICZ \& KAMIL (1981).

Such short-term effects can have a potent influence on the pattern of food selection. In an experiment in which pigeons searched for familiar, natural food grains against a complex gravel background, BOND (1983) found clear evidence of sustained over-selection of the more common grain type. The results implicated a perceptual process, rather than simply a change in preference, since they were evident only when the grains were presented on a natural, cryptic background. When the same grains were shown on a uniform gray background, on which they were all highly conspicuous, the over-selection effect was not observed.

One clue to the nature of the underlying perceptual mechanism can be found in the evidence that animals in such "free choice" preparations, in which many different target stimuli are presented simultaneously, tend to respond in nonrandom sequences, consisting of "runs" of a single target type (DAWKINS 1971b; BOND 1982). Such results suggest that a similar perceptual change could be induced by presenting the stimuli one at a time and manipulating the imposed sequence of stimuli to produce a run of a particular stimulus type.

This approach transforms visual search for multiple food types into a standard serial detection task. The animal is presented with a series of visual displays. Some of these are positives, containing one or more target stimuli interspersed with elements of the background; the others are negatives, containing only background items. The animal has two response options: "Positive," indicating that a target stimulus was detected in the display, or "Negative," indicating that no target stimulus was discovered.

Using such a serial detection task, PIETREWICZ \& Kamil $(1977,1979)$ trained blue jays to detect cryptic moths in back-projected slide images. They found that runs in the imposed stimulus sequence resulted in a significant improvement in the probability of detection. Similar results have been obtained with pigeons searching for letters on a computer display (BLOUGH 1989) and have commonly been observed in investigations of the "repetition effect" in human visual-choice tasks (RABBITT et al. 1977). 
The central concern in the study of searching images thus devolves on the mechanism of the "Run Effect." Is performance on a serial-detection task enhanced by exposure to runs of a single positive stimulus type? How large is the enhancement? Under what circumstances does it occur? What are the effects of a run on subsequent performance on negatives or positives of other types? What can we infer from such results about the underlying cognitive process?

\section{Three Alternative Hypotheses}

\section{Discriminability}

Three principal alternative mechanisms could account for the Run Effect. The first is the Discriminability Hypothesis, which postulates a change in the ability to detect the stimulus. In signal-detection theory, "discriminability," or d', is a measure of the sensitivity of an observer, an estimate of his ability to correctly identify a stimulus as either a positive or a negative (GREEN \& SWETS 1966; GETTY et al. 1987). This first hypothesis is, therefore, close to TINBERGEN's (1960) original conception of searching image as a transitory improvement in perceptual ability.

In the context of a serial-detection task, the most likely mechanism for an increase in discriminability is "attentional priming" (BLOUGH 1989), in which advanced information about a succeeding stimulus modifies an attentional mechanism. A strong form of the Discriminability Hypothesis thus asserts that when the observer is primed to expect or anticipate a particular stimulus, he requires less information to detect it. Attention to a particular type of stimulus will, therefore, increase the accuracy of detection of that stimulus and/or decrease the response time.

An increase in the detectability of one type of stimulus, if it results from selective attention, has commonly been assumed to occur only at the cost of overlooking other, alternative stimuli (SUTHERLAND \& MACKINTOSH 1971; RILEY \& LEITH 1976). This "Inverse Effect" is a characteristic feature of selective attention in humans (KAHNEMAN 1973) and has, therefore, been proposed as the operationally defining feature of an attentional process (MACKINTOSH 1975). It has proved to be exceedingly difficult to confirm with animal subjects, though there is some evidence for it from matching-to-sample experiments using simple, artificial stimuli (LAMB 1983; BROWN et al. 1984). The Inverse Effect thus constitutes an ancillary issue within the Discriminability Hypothesis: Is an enhancement of detection of the stimulus within a run necessarily accompanied by a reduction in the detectability of other stimulus types?

\section{Response Bias}

An alternative to a change in discriminability is the Response Bias Hypothesis. An improvement in performance following a run, in this view, is the result, not of changes in the animal's perceptual ability, but of changes in its predisposition to respond "Positive." The positive stimulus will have been encountered more frequently than usual in the course of a run. Correct responses to these stimuli will, therefore, have been rewarded more often. As a result of the increase in reward frequency, the subsequent probability of responding "Positive" will be enhanced. 
In a free-choice experiment with multiple food types, a response bias would be exhibited as a transitory preference for one food type over others (BOND 1983). In a serial detection task, where the subject must make a distinctive response to negative, as well as to positive stimuli, a response bias would be exhibited as a shift in what signal-detection theory terms the "criterion." The criterion, $\boldsymbol{\beta}$, is a measure of the predisposition of the subject to respond "Positive" to any stimulus presented (GREEN \& SWETS 1966).

When $\boldsymbol{\beta}$ is equal to 1.0 , the subject's responses are equally balanced between responding "Positive" and "Negative." A value less than 1.0 indicates a bias toward responding "Negative"; correspondingly, a value greater than 1.0 indicates a bias toward responding "Positive." If frequent encounters with positive stimuli cause the criterion to shift toward higher values, performance on positives will improve, albeit at the expense of increased numbers of erroneous "Positive" responses to negative stimuli.

\section{Caution}

The third alternative hypothesis derives from the fact that the amount of time that the subject has available for examining each stimulus is not fixed. In serialdetection tasks, the stimulus is displayed continuously until the subject makes a response; in a free-choice design, the rate at which the subject scans the stimulus display is uncontrolled. Under these circumstances, an additional degree of freedom is introduced: The subject has the option of increasing the viewing time for the stimulus, and thereby improving his accuracy, or decreasing the viewing time, and thereby improving his speed. This is the speed-accuracy trade-off.

The speed-accuracy trade-off is best considered as the consequence of a third parameter, what VICKERS (1979) has called the "caution" of the subject. A cautious subject will search a stimulus display with great thoroughness before responding; an incautious subject will respond rapidly, on the basis of less complete information. The Caution Hypothesis, thus, attributes improvements in performance to changes in the amount of evidence that the subject acquires before making a stimulus categorization. An increase in caution results in an increase in d', thereby mimicking a change in discriminability, but it does so by virtue of increasing the response time or slowing the rate of search.

The significance of the caution of a visual search has recently been emphasized by a number of researchers. GETTY et al. (1987) have observed that blue jays searching for images of cryptic moths may show changes in either discriminability or search rate and have suggested that individual birds may vary in the strategy they adopt. GENDRON \& STADDON $(1983,1984)$ have developed models for predicting the optimal searching rate for a visual predator. GENDRON (1986) subsequently tested several of these predictions, using bobwhite quail and found indications of changes in both search rate and discriminability. GENDRON's (1986) results, in turn, inspired GUILFORD \& DAWKINS (1987) to argue that none of the existing studies in the searching-image literature had unequivocally eliminated variation in searching rate as an alternative hypothesis.

The argument for how changes in caution might account for a Run Effect is, roughly, as follows: When positive stimuli differ in crypticity, they will also differ in 
the levels of caution that will maximize the rate of their discovery (GENDRON \& STADDON 1983). When two such stimulus types are presented in a mixed sequence, the subject will adopt a searching rate that represents a compromise between their respective optima. The resulting rate will be too slow for maximum detection efficiency on the more conspicuous stimulus and too fast for the more cryptic one (GENDRON \& STADDON 1983).

The predicted effects of a run of positives would then be asymmetrical, depending on which stimulus type was used in the run (GUILFORD \& DAWKINS 1987). A run of the cryptic type would induce an increase in caution, reducing the search rate and increasing the detection accuracy within the run. Detection of succeeding positives of the opposite type would be relatively unaffected. A run of the more conspicuous type would cause a reduction in caution, increasing the search rate within the run and decreasing the detection accuracy for any subsequent positives of the less conspicuous type.

All three hypotheses - Discriminability, Response Bias, and Caution - thus seem capable of producing, under some circumstances, a transitory increase in correct responses to positive stimuli as a result of a run in the imposed stimulus sequence. Several other studies have addressed, at least inferentially, one or another of the alternatives (PIETREWICZ \& KAMIL 1979, 1981; BOND 1983; GENDRON 1986; BLOUGH 1989). An explicit test of their relative strengths has never been performed, however.

To distinguish among the three alternatives, we will present a single, coherent model, one that includes discriminability, response bias, and caution as specific, independent components. The consequences of varying each parameter will then be evaluated objectively by computer simulation, generating predicted effects on response time and accuracy for each stimulus type. A subsequent experimental test of the hypotheses will then be conducted, using pigeons searching for images of cryptic food grains and comparing their behavior to the predictions of the model.

\section{The Accumulator Model}

Visual discrimination has often been conceived of as the outcome of sequential stimulus sampling (SMITH \& VICKERS 1988). The flow of perception is viewed in these models as a series of discrete observations, constituting successive samples of values from a stimulus display. For simplicity, it is commonly assumed that each observation provides a constant amount of information and takes a constant amount of time. The evidence provided by the observations is continuously cumulated, in some fashion, and the final response is determined on the basis of the cumulative total.

One of the most successful of the sequential sampling models is the "accumulator" model, which VICKERS (1970) developed as a way of accounting for several features of response time and accuracy in visual discrimination experiments. In particular, when the difficulty of a discrimination task is varied from trial to trial, subjects invariably make more errors on more difficult discrimination, as one might expect (VICKERS 1979). The response time also varies in these experiments, however, and in a fully correlated fashion: Subjects make more errors on the more difficult discriminations, and they take longer to make them (VICKERS 1979). 
These results suggested that speed and accuracy are aspects of a single, integrated decision process. VICKERS (1970) undertook to model this process through an adaptation of the sequential-sampling paradigm. His approach assumes that the evidence provided by the sequential observations is summed in competing "accumulators," with one accumulator for each of the possible response categories (i.e. "Positive" or "Negative"). Although the observations are discrete, the evidentiary values in this model are continuous, with larger values being generated by observations that are more consistent with a given stimulus type. When the total evidence in favor of any one of the possible outcomes exceeds some specified threshold, an appropriate response is made.

The accumulator model readily accounts for the observed relationship between speed and accuracy in discrimination trials that vary in task difficulty. In a trial with a conspicuous target that bears little resemblance to the surrounding background, each observation will be fairly unequivocal, implicating only the correct response alternative, and few of them will be added into the wrong accumulator. The result will be responses that are both fast and accurate.

On the other hand, in a trial with a target stimulus that resembles the background in some respects, there will be many ambiguous or misleading observations, and errors will be made in assigning them to the appropriate accumulator. More observations will therefore be needed to fill the correct accumulator up to the threshold value, thus increasing the response time. In addition, the possibility of obtaining enough mistaken observations to trigger the threshold on the wrong accumulator is increased. The result will be responses that are both slower and less accurate.

The attractions of the accumulator model are not limited to its explanation of this one effect, however. As VICKERS (1979) has shown, this deceptively simple algorithm appears to account for a remarkable number of features of a variety of different experiments in visual perception. It therefore seemed worthwhile to formulate a version of the accumulator model that would be applicable to visual search for cryptic targets and to use it as a means fur evaluating the explanatory strength of the three hypotheses.

\section{Simulation Methods}

As in VICKERS' (1979) accumulator model of signal detection, our algorithm used two accumulators, one for "Positive" and one for "Negative" responses, each of them controlled by separate threshold variables. Targets and backgrounds were described in terms of their characteristic distribution along a single dimension of "stimulus value" (Fig. 1A). A detection trial consisted of a series of samples from these underlying stimulus distributions. If the sample accorded more closely with the hypothesis that it originated from the background distribution, its value was added to the "Negative" accumulator; if it accorded more closely with a target distribution, it was added to the "Positive" accumulator.

In our model, however, the algorithm searched for two cryptic target types simultaneously, and variance in the stimulus value of the background was assumed to be much larger than that of either of the targets (Fig. 1B). During negative trials, all samples originated from the background distribution (Fig. 1A). During positive trials, however, some samples came from the background distribution and some from that of one of the targets (Fig. 1B). A weighting parameter, termed the "salience" of the target, 
Fig. 1: Examples of distributions of stimulus value $(V)$ used as input to the accumulator model. A) Negative trial, with $\bar{X}=0, S D=$ 1. B) Positive trial, exhibiting a conjunct distribution. Stimulus values for the background are the same as in A; stimulus values for the target are drawn from a narrower distribution, with $\bar{X}=1.0, S D=0.1$. The relative likelihood of sampling from the background or the target is determined by the salience parameter, $S$, indicated by the relative areas under the two curves. C) Example of distributions for two disparate targets: $\mathrm{A}=$ cryptic target type, with $\bar{X}=-0.8, \mathrm{~B}=$ more conspicuous target, with $\bar{X}=1.0$. Even this small a difference in relative crypticity can have striking effects on accuracy and response time. Dashed curve: background distribution.
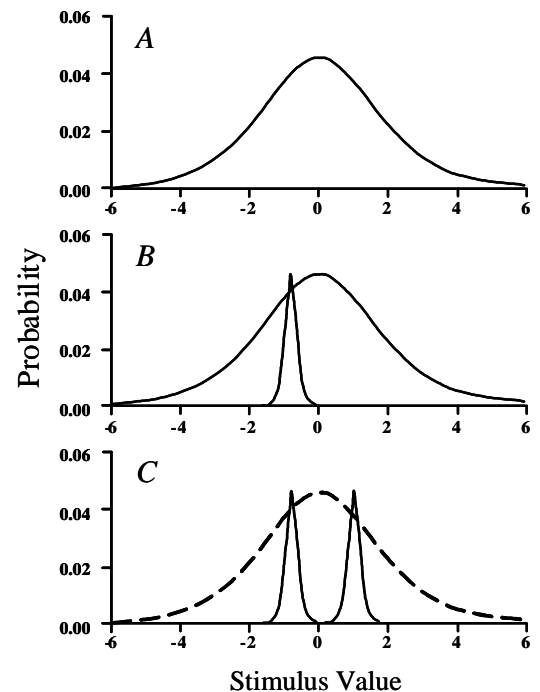

was used to determine whether any given sample in a positive trial would come from the target or the background distribution (Fig. 1B).

The principal independent variable in our simulations was the relative crypticity of the two target types. In no case did the pair of targets bear any substantial resemblance to one another, in that their distributions of stimulus value did not overlap (Fig. 1C). Their relative crypticity was manipulated by shifting the means of the target distributions, thereby modifying their degree of resemblance to the background (Fig. 1C). All possible combinations of relatively conspicuous and relatively cryptic targets were used.

For each pair of target crypticities, we first computed optimal values for the two accumulator thresholds, with an optimization procedure that gave equal weight to speed and accuracy. The results of the optimization provided a baseline indication of the pattern of responses to a particular combination of target crypticities. We then manipulated the accumulator thresholds around their computed optima to simulate the effects of changes in response bias and caution. To simulate changes in crypticity, we also manipulated the relative salience of the targets. A full description of the algorithm and simulation methods is provided in Appendix A.

\section{Simulation Results}

In all simulations, "Negative" responses, whether to positive or negative stimuli, were slower than "Positive" ones. This might have been expected, since "Negative" responses generally result from a more exhaustive search. In addition, however, correct responses were invariably faster than erroneous responses of the same type: "Positive" responses to positives (i.e. "hits") were faster than "Positive" responses to negatives (i.e. "false alarms"). Likewise, "Negative" responses to negatives (i.e. "correct rejections") were faster than "Negative" responses to positives (i.e. "misses"). VICKERS (1979) reported this to be a characteristic feature of the accumulator model, 
one that it shares with no other existing model of visual perception. He noted that similar response-time effects have commonly been observed in visual discrimination studies in which the subject is instructed to be as fast and as accurate as possible.

When the accumulator thresholds were optimized, accuracy on the two types of positive targets was strikingly asymmetrical. This was true even when the targets exhibited similar degrees of relative crypticity, that is, when the means of their distributions were similar distances from the mean of the background. With only a $6 \%$ difference in crypticity, for example, accuracy on the more conspicuous target was $92 \%$, while that on the more cryptic one was $78 \%$. The disparity was only slightly larger - $100 \%$ versus $73 \%$ - with an $88 \%$ difference in crypticity. Response time was much more sensitive than accuracy to the relative crypticity of the targets: where the difference in crypticity was $6 \%$, the more cryptic target took $12 \%$ longer to discover; where the difference was $88 \%$, responses to the more cryptic target took twice as long. The results suggest that the effects of small differences in crypticity may be greatly exaggerated when animals are attempting to maximize their rate of food discovery. Asymmetries in detection accuracy in these situations may be unavoidable.

\section{Predicted Effects}

Predicted directions and magnitudes of the effects of a run of positives were derived from the simulation experiments (Table 1). In general, the Response-Bias Hypothesis predicts that accuracy will increase and correct response time fall for both positive types following a run of either type. The effect may be weaker for more conspicuous positives, if performance is already close to maximum. This improvement in performance on positives is at the expense of that on negatives: accuracy on negative trials will decrease and correct response time for negatives will increase. Error response times for positives will increase, while those for negatives will remain roughly constant.

The Caution Hypothesis makes different predictions depending on the relative crypticity of the positive stimulus used in the run. A run of more cryptic positives should increase caution, while that of more conspicuous positives should decrease it. The strongest and most consistent effect of increased caution is to increase correct response time for negatives and error response time for all stimulus types. Increases in caution also result in increased accuracy on positives, at the expense of an increase in correct response time. Decreased caution has the opposite effect, decreasing response time for all types of correct responses, as well as errors, and decreasing accuracy on positives. The effects of decreases in caution were generally more striking than those of increases.

The Discriminability Hypothesis predicts that accuracy will increase and correct response time fall for the positive stimulus in the run. Accuracy and correct response time for negatives will be unaffected, but error response time for negatives may increase slightly. There may be some decrease in accuracy on the opposite positive stimulus, even if it does not actually suffer a compensatory decrease in 
Table 1: Predictions of the three hypotheses, derived from simulations of the Accumulator Model. Positives are of two types: relatively cryptic and relatively conspicuous. Dependent variables are \% correct responses (PC), median response time for correct responses (RTC), and median response time for erroneous responses (RTE). +: higher levels of the given variable during or following a run of positives of the specified type, -; lower levels. Where the effect depended on the choice of initial stimulus parameters, or was comparatively small, the resulting uncertainty is indicated.

\begin{tabular}{|c|c|c|c|c|c|c|}
\hline \multirow[b]{4}{*}{ Var } & \multirow[b]{4}{*}{ Stimulus } & \multicolumn{5}{|c|}{ Hypotheses } \\
\hline & & \multirow{3}{*}{$\begin{array}{c}\text { Response } \\
\text { Bias }\end{array}$} & \multicolumn{2}{|c|}{ Caution } & \multicolumn{2}{|c|}{ Discriminability } \\
\hline & & & Run of & Run of & Run of & Run of \\
\hline & & & Cryptic & Conspic & Cryptic & Conspic \\
\hline \multirow[t]{3}{*}{$\mathrm{PC}$} & $\mathrm{Neg}$ & - & $-I=$ & $+/=$ & $=$ & $=$ \\
\hline & Cryptic Pos & + & + & - & + & - \\
\hline & Conspic Pos & $+1=$ & $+1=$ & $=$ & $-I=$ & + \\
\hline \multirow[t]{3}{*}{$\mathrm{RT}_{\mathrm{C}}$} & $\mathrm{Neg}$ & + & + & - & $=$ & $=$ \\
\hline & Cryptic Pos & - & $+1=$ & - & - & + \\
\hline & Conspic Pos & $-1=$ & $+1=$ & - & $+1=$ & $-1=$ \\
\hline \multirow[t]{3}{*}{$\mathrm{RT}_{\mathrm{E}}$} & $\mathrm{Neg}$ & $=$ & + & - & $+1=$ & $+1=$ \\
\hline & Cryptic Pos & + & + & - & $=$ & $=$ \\
\hline & Conspic Pos & + & + & - & $=$ & $=$ \\
\hline
\end{tabular}

discriminability. if an additional Inverse Effect is explicitly added to the model, with discriminability declining for the opposite positive type as well as increasing for the run positive, the effect on the opposite positive is an unequivocal decrease in accuracy and increase in correct response time. Error response times to positives will not be affected in either case.

An Inverse Effect is also rioted when the accumulator thresholds are optimized subsequent to increasing the discriminability of the positive in the run. This manipulation, which simulates a change in both discriminability and caution, has differing effects, depending on the initial relative crypticity of the two stimuli and on which stimulus was used in the run. Increasing the discriminability of a cryptic positive has proportionately more of an impact on accuracy than on response time, so the subsequent optimization tends to compensate by decreasing caution. Increasing that of a more conspicuous positive, on the other hand, has more of an impact on response time, because accuracy in responding to the conspicuous stimulus is already close to maximum. Optimization in this case increases caution, because the model tries to compensate for the excessive speed by making changes that increase accuracy.

In either case, however, the result of the change in caution is to decrease performance on the opposite positive type. When it is the cryptic stimulus whose discriminability is enhanced, response times for both positive types decrease after optimization. Accuracy for the inure cryptic positive increases, but that for the opposite, more conspicuous positive declines by as much as $10 \%$. Increasing the discriminability of the conspicuous stimulus, on the other hand, causes response times for conspicuous positives to drop by as much as $30 \%$ after optimization. For the opposite, 
more cryptic positive, however, accuracy invariably decreases and response time increases, often by substantial amounts. These results suggest that compensatory changes in caution, when combined with an increase in discriminability for the run positive, can generate an Inverse Effect, even when the discriminability of the opposite positive is actually unaltered.

The predicted effects of the three hypotheses were tested on pigeons in a serialdetection task involving back-projected slides of natural stimuli, similar to the protocol developed by PIETREWICZ \& KAMIL (1979). Using the same food grains and gravel backgrounds that were effective in producing a selection bias in the free-choice study (BOND 1983), we generated slides containing either gravel alone or gravel with a single-stimulus grain and trained pigeons to search for the grain positives. Responses to stimuli during and following sequences of runs of a single positive stimulus type were then compared to control sequences containing mixtures of negatives and of positives of both types to detect changes in accuracy and response time.

\section{Methods}

\section{Stimuli}

Backgrounds were prepared by coating plywood panels with a mixture of beach gravel and casting resin (BOND 1983). To provide a second background type against which both grains would he readily discriminable, we subsequently spray-painted several of the gravel panels with gray enamel. Black beans (Phaseolus mungo L.) and red wheat (Triticum durum Desf.), which had elicited the strongest switching effects in the free-choice study (BOND 1983), were placed individually on the backgrounds and photographed with fine-grained color film. A light table was used to obtain uniform illumination, and the camera was fitted with a polarizing filter to minimize specular reflectance.

On the natural, "mixed" background, positive stimuli contained a single grain of either wheat or beans in one of the four quadrants of the slide; negative stimuli contained only gravel. On the gray background, one additional, naturally colored object was placed in each quadrant of the slide. For negative stimuli, all four objects were pieces of plastic-coated gravel; for positives, one of the four objects was a grain, either a bean or a wheat seed, and the other three were pieces of gravel. The background was shifted systematically between successive shots, so that the same gravel configuration was never exhibited in any two slides, and no particular grain was ever photographed twice.

\section{Apparatus}

Slide images were back-projected on a ground-glass screen, using two Kodak Ektagraphic carousel projectors equipped with high-speed shutters. The effective magnification of the projected image was about 1.5 times natural size in the experimental trials. A higher magnification was used during training.

The screen was mounted behind a $7.5-\mathrm{cm}^{2}$ window in the back wall of an operant chamber. Four pigeon keys of transparent lucite divided the image on the screen into quadrants. A circular advance key, $2.5 \mathrm{~cm}$ in diameter, was placed $10 \mathrm{~cm}$ to the left of the center of the fourkey array. Whenever a slide was shown on the screen, the advance key was illuminated in red. A grain hopper was centered $8.5 \mathrm{~cm}$ below the four-key array. All apparatus was controlled by a microprocessor interfaced with a DEC PDP-11/23 minicomputer. 
Each stimulus set consisted of 40 positives of each grain type and 80 negatives. To maintain variability, we prepared ten additional positive slides of each grain type and twenty additional negatives and periodically interchanged them with the experimental stimuli. Positives and negatives were distributed in alternating positions in the two carousels to minimize the interval between trials and to prevent the sound of the projector advance from being used as a cue to the contents of the slide. Since the photographs were taken under uniform illumination, the slides lacked implicit directional cues. They could, therefore, be rotated or reversed into any of eight different orientations. To reduce the birds' tendency to solve the task by memorizing individual stimuli, we randomized the order and orientation of all slides before every fifth session.

\section{Subjects}

The subjects, three experimentally naive White Carneaux pigeons, were maintained in individual cages at 75-80\% of their free-feeding weight. Their daily food ration included substantial proportions of buds of the stimulus-grain types, so they were thoroughly familiar with the stimuli prior to beginning training. The birds were first conditioned to peck the fourkey array and the advance key. They were then trained on three progressively more difficult discrimination tasks.

Training was conducted in sessions of 200 trials per day, beginning with the graybackground stimulus set. When this task had been acquired, the birds were transferred to mixedbackground stimuli, first at high magnification $(3: 1)$ and then at low $(1.5: 1)$. The criterion for having learned a task was at least $80 \%$ correct responses to both positives and negatives for five consecutive sessions. The full training period, from initial conditioning to commencement of experimental trials, required about four months.

\section{Procedure}

The sequence of events during a trial was as follows: Following an intertrial interval, the screen was uniformly illuminated in yellow to indicate the start of a new trial. This warning signal was sustained until the bird pecked the display. After a delay of $100 \mathrm{~ms}$, a stimulus slide was displayed, and the red advance key was illuminated. On all trials, a single peck to the advance key, or to any of the keys in the four-key array, darkened both the screen and the advance key and terminated the trial. The computer recorded which stimulus type had been displayed, what the bird's response was, and how long it took to make the response. It then issued an appropriate reward.

If the stimulus shown was a positive, a peck to the quadrant of the screen containing the grain was rewarded with $1.5 \mathrm{~s}$ of access to food; if the stimulus was a negative, a peck to the advance key (i.e. a correct rejection) was rewarded, $25 \%$ of the time on a random basis, with a similar food access. This rate of partial reinforcement for negatives was determined empirically to produce stable performance with a fairly strict criterion (i.e. a minimum frequency of false alarms). Pecks to quadrants containing only gravel were never rewarded.

The experimental unit was a session of 32 trials, divided into four blocks (Fig. 2). The Control Block consisted of 12 trials - 6 negatives, 3 bean positives, and 3 wheat positives block-randomized in groups of 4 . The second block was a string of 6 consecutive negatives. It was followed by the Treatment Block, consisting of a string of 6 consecutive positives, all of the same grain type. Finally, the Assay Block was a set of 8 trials, 4 positives and 4 negatives block-randomized in pairs. Again, all positives in the block were of the same grain type.

On half of the sessions, the Assay Block used positives of the same type as the Treatment Block; in the remainder, the opposite grain type was used. There were, therefore, four types of session: Bean Treatment / Bean Assay; Bean Treatment / Wheat Assay; Wheat Treatment / Wheat Assay; and Wheat Treatment / Bean Assay. Birds were presented with eight sessions per day, two of each session type, block- 


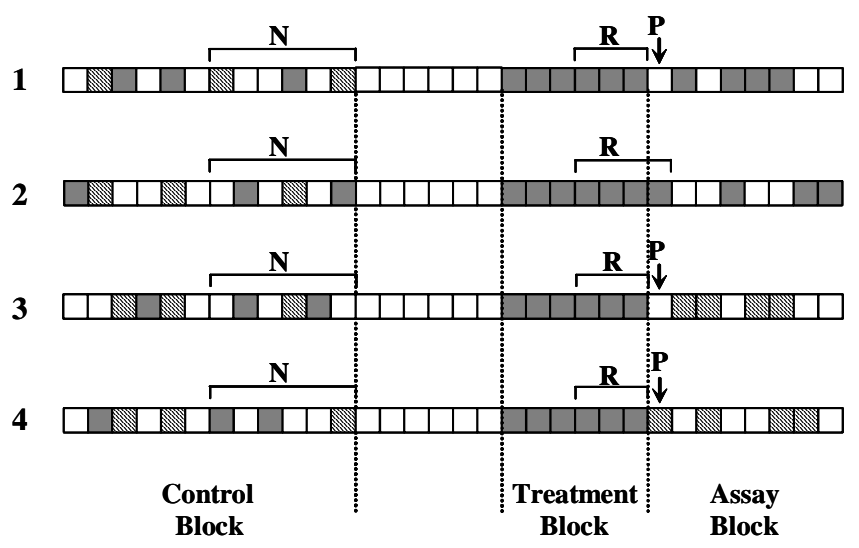

Fig. 2: Diagram of four typical experimental sessions. Each square represents a trial, with trial sequence proceeding from left to right. Blank squares: negative slides, lined squares: wheat positives, cross-hatched squares: beans. $\mathbf{N}$ : non-runs sample, consisting of the last six trials of the Control Block. In all of these sessions, the Treatment Block is shown as a run of six successive bean positives. In sessions 1, 3, and 4, the within-run sample (R) consisted of the last three trials of the Treatment Block; in session 2, this sample also incorporated the first trial of the following Assay Block, because it was of the same type as the Treatment Block. Probe samples $(\mathbf{P})$ of negatives and wheat positives following the Treatment Block of beans were obtained from the first trial in the Assay Block in sessions 1,3 , and 4

randomized in sets of four. There were no delays between successive sessions. The initial experiment consisted of 50 days of this regimen using stimuli with mixed-gravel backgrounds. Following a brief retraining period, the experiment was then replicated using gray background slides. The resulting data set included 100 sessions of each treatment/assay combination on each background for each bird.

\section{Results}

\section{Design of the Analysis}

The analysis focused on three issues: the changes in performance in the course of a run of positives of a single grain type, and the effects of the positive run on subsequent performance on negative stimuli and on positives of the opposite type. The underlying design, thus, was a two-way factorial experiment, with one factor being stimulus type (Negative, Bean, or Wheat) and the other being treatment (Non-run, consisting of trials from a mixed sequence of negatives and positives of both types; Bean Run, consisting of trials within or following a Treatment Block of bean positives; or Wheat Run, consisting of trials within or following a Treatment Block of 
wheat). The effects of interest were contrasts for each stimulus type between the nonrun treatment and each of the run treatments.

The non-run sample consisted of the last six trials of each Control Block ( $\mathbf{N}$ in Fig. 2). Because performance during the Treatment Block was generally stable after the second trial, the last three trials of each Treatment Block were taken as the best indication of the change in performance within a run ( $\mathbf{R}$ in Fig. 2). When the first trial of the Assay Block was the same type of positive as the preceding Treatment Block, it was also included in the within-run sample (Fig. 2, Session 2). These constituted the sample for the Bean Stimulus / Bean Run and Wheat Stimulus / Wheat Run cells of the design.

The Assay Block was intended to characterize effects of the run treatment that might persist across a series of subsequent trials. There was, however, no evidence of a difference from the non-run sample for anything beyond the initial trial of the Assay Block. The issues of the effect of the run treatment on performance on negatives and on positives of the opposite type were therefore addressed using only the first trial of each of the appropriate Assay Blocks. These "Probe" trials provided the samples for the remaining cells of the design (P in Fig. 2).

Fig. 3: Treatment effects on mixed background. Upward-pointing triangles: measure was greater for the indicated run treatment than for the comparable non-run treatment, downward triangles: reduction in the measure. Filled symbols: significant difference $(p<0.05)$, open symbols: non-significant trend $(p<0.07)$.

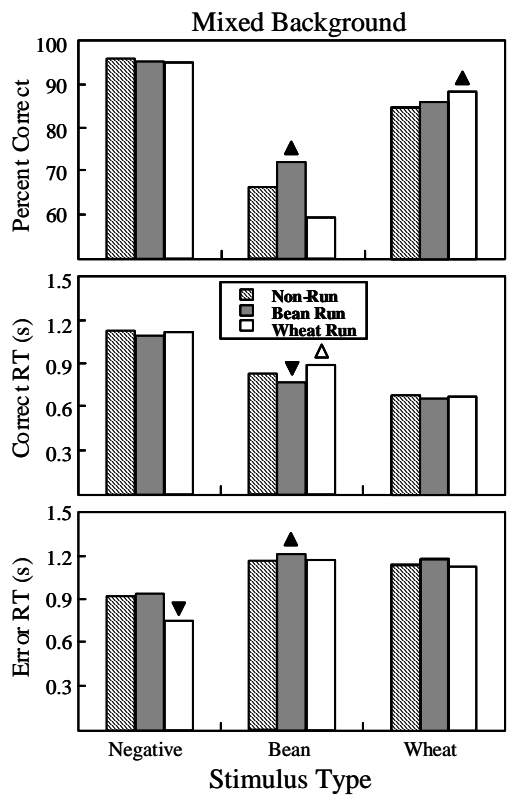

Trials were pooled across sessions within subjects. On each background, the non-run samples contained roughly 1200 negative trials and 600 positives of each type for each bird. Sample sizes within runs were about 600 trials, while those for negative probes and positive probes were about 100 and 50, respectively. Three measures of performance were obtained for each treatment combination for each bird: accuracy, as percentage of correct responses; median response time for correct responses; and median response time for error responses (Figs. 3, 4). 
Fig. 4: Treatment effects on gray background. Upward-pointing triangles: measure was greater for the indicated run treatment than for the comparable non-run treatment, downward triangles: reduction in the measure. Filled symbols: significant difference $(p$ $<0.05)$, open symbols: nonsignificant trend $(p<0.07)$.

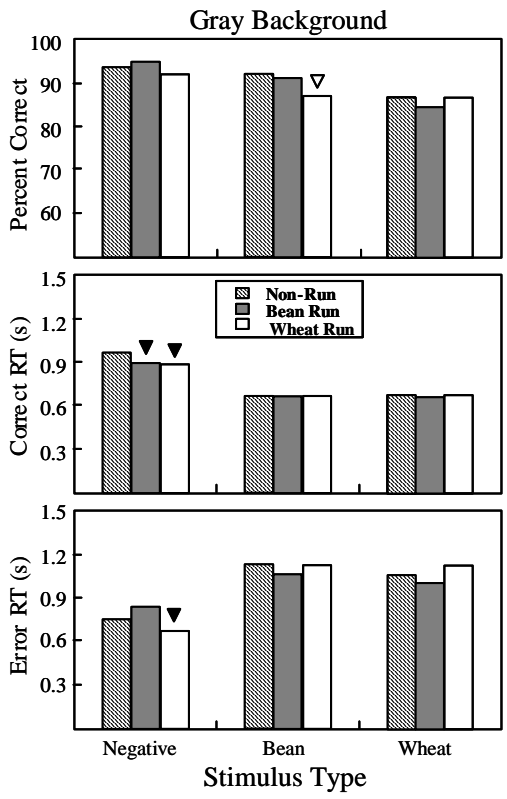

\section{Over-All Patterns}

Wheat appeared to be more conspicuous than beans on the mixed background, in that accuracy on wheat was consistently higher and correct response time consistently faster. This was true for all birds across all treatments. The difference in accuracy was striking, ranging from 11 to 23 percentage points. The difference in response time was consistently about $200 \mathrm{~ms}$.

This difference between the positive stimuli was reversed on the gray background, where beans were responded to faster and more accurately than wheat. The differences between stimuli in this task were, however, generally smaller. The accuracy difference was no more than 10 percentage points for any bird, while the response-time difference was on the order of 10 to $30 \mathrm{~ms}$.

Accuracy on negatives was invariably superior to that on positives and was never less than $93 \%$ for any bird on either background. This suggests a relatively strict signal-detection criterion, disproportionately reducing the occurrence of false alarms. "Positive" responses (i.e. hits and false alarms) were invariably faster than "Negative" responses (i.e. correct rejections and misses) for all birds across all treatments. Correct responses were also faster than erroneous responses of the same type: hits were invariably faster than false alarms; correct rejections were faster than misses. All of these response-time effects were predicted by the accumulator model, lending credence to its use in evaluating the three hypothetical mechanisms.

\section{Treatment Effects on Accuracy}

Analysis of accuracy was conducted on the raw totals of correct and incorrect responses on each background using linear modeling (GRIZZLE et al. 1969), which is an analog to analysis of variance for categorical data. Responses were classified accord- 
ing to stimulus type, treatment type, and correctness (correct vs. wrong), resulting in a $3 \times 3 \times 2$ contingency table for each bird. The effects of interest were explicit contrasts between the run and non-run conditions for each combination of stimulus type and run type. Analysis was conducted on each bird individually, as well as on an aggregate model that included subjects as an additional dimension, using the SAS Catmod package (SAS 1985). Results of the aggregate analysis are summarized in the upper panels of Figs. 3 and 4.

Accuracy on wheat and beans increased significantly during runs on the mixed background $(p<0.003)$, but not on the gray. There were indications of a reduction in accuracy on beans following a wheat run: two birds showed trends in this direction ( $p$ $<0.07$ ) on the mixed background, and one bird exhibited a similar trend on the gray. The difference was generally large, however, roughly twice the size of the increases seen during bean runs, suggesting that the low levels of significance reflect the much smaller sample sizes involved in this treatment combination. Accuracy on wheat following a bean run was virtually unaffected. Neither of the run treatments had any consistent effect on accuracy on negative stimuli on either of the backgrounds, though one bird showed a significant decrease in accuracy following a wheat run on the gray background.

\section{Treatment Effects on Response Time}

Analysis of response time was conducted separately for correct and erroneous responses. We used Wilcoxon rank tests to contrast the survival distributions for run and non-run conditions for each combination of stimulus type and run type, using the SAS Lifetest package (SAS 1985). Analyses of individual birds were performed, as well as aggregate analyses that were stratified by subject. Results of the aggregate analyses are summarized in the lower two panels on Figs. 3 and 4.

Correct response times for negatives were unaffected by either run treatment on the mixed background, but decreased following a run of positives of either type on the gray $(p<0.03)$. On the mixed background, correct responses were significantly faster during a bean run $(p<0.001)$, but were not consistently accelerated during wheat runs. Similarly, correct responses to beans might have been slower following a wheat run ( $p$ $<0.08)$, but responses to wheat were clearly not affected by bean runs $(p>0.2)$. On the gray background, correct response times for positive stimuli were unaffected by either treatment type.

Error-response times were shorter for negatives following a wheat run on both the mixed $(p<0.05)$ and gray $(p<0.001)$ backgrounds, but bean runs had no significant impact on error responses to negatives $(p>0.3)$. On the mixed background, erroneous responses to beans were slower during and following a bean run $(p<0.03)$. Error-response times to either positive type were not affected by wheat runs on either background. 


\section{Discussion}

\section{Evaluation of the Three Hypotheses}

On the mixed background, runs of a single positive stimulus type resulted in significant improvements in performance (Fig. 3). Accuracy increased and correct response time decreased for beans during a run. This effect seemed to be reversed for bean probes following a run of wheat, where trends to a reduction in accuracy and an increase in correct response time were observed. Accuracy increased during a run of wheat, but correct response time was unaffected. Neither accuracy nor correct response time for negatives was modified following either run treatment.

Given that beans were clearly less conspicuous than wheat on the mixed background, these findings conform to the predictions of the Discriminability Hypothesis (Table 1). In contrast, neither response bias nor caution seems able fully to account for the effects. Contrary to the Response Bias Hypothesis, changes in accuracy and correct response time were not invariably in the same direction for both stimulus types: for example, accuracy for wheat increased during a run of wheat, but accuracy for beans following a run of wheat did not increase, and may actually have declined (Fig. 3).

Contrary to the Caution Hypothesis, response times did not invariably increase during and following a bean run and decrease during and following a wheat run. In fact, correct response times dropped significantly in the course of a bean run and showed an increasing trend for beans following a wheat run (Fig. 3). The significant increase in accuracy on wheat in the course of a wheat run is also contrary to the Caution Hypothesis, which predicts effects on response time, rather than accuracy, for this treatment combination.

The most compelling evidence in favor of the Discriminability Hypothesis, however, is the absence of any indication of a decline in performance on negatives with either run treatment. This contradicts both the Caution and the Response Bias Hypotheses: If there were no change in the bird's ability to detect the image of a grain in the positive slides, improvement in performance on positive stimuli could only be obtained at the expense of some degradation in performance on negatives. This was an invariant feature of simulations that manipulated caution and response bias (Table 1), and it was not observed to occur in the experiment (Fig. 3).

Additional evidence in support of a discriminability effect is provided by the fact that enhancement of performance within runs was not observed in the gray background stimuli (Fig. 4). A comparable dependence on background type was observed in the free-choice experiment (Bond 1983). Shifts in selective attention ought to be more influential when the discrimination task is more difficult and more information must be processed before making a decision (RILEY \& ROITBLAT 1978). To the extent that changes in discriminability result from attentional priming (BLOUGH 1989), therefore, the Discriminability Hypothesis predicts smaller effects with more conspicuous stimuli, exactly as we have observed. In contrast, the Caution Hypothesis assumes that one stimulus will be more conspicuous than the other, but does not require that both of them be cryptic, while the Response Bias Hypothesis should operate irrespective of stimulus crypticity. 
Caution and the Inverse Effect

The experimental evidence, thus, is strongly in favor of the Discriminability Hypothesis. In this regard, our results support TINBERGEN's (1960) original, perceptual account of the searching-image phenomenon and are in agreement with other studies of the effect of runs in a serial detection task (PIETREWICZ \& KAMIL 1979, 1981; BLOUGH 1989). However, changes in caution may also be occurring in the course of a run of positives.

On the mixed background, there was a significant increase in the error-response time for beans in the course of a bean run and a decrease in that for negatives following a wheat run (Fig. 3). Neither of these effects can be explained by the Discriminability Hypothesis, which predicts no change in error response times (Table I). They are, however, both in the direction of changes predicted by the Caution Hypothesis. The significant reductions in response time observed to accompany positive runs on the gray background (Fig. 4) also suggest a reduction in caution.

If changes are occurring in both caution and discriminability, the Accumulator Model offers a possible explanation. If the imposition of a run of a single type of positive improves the discriminability of that stimulus, it will also shift the optimum values for the accumulator thresholds. If the animal then compensates by changing its thresholds in the direction of optimality, it will almost necessarily change the level of caution, as well.

When this combination of influences was simulated, the improvement in detection for the stimulus in the run was invariably accompanied by an Inverse Effect, a decrease in performance on positives of the opposite type, even when the discriminability of the opposite stimulus was kept constant. The magnitude of the Inverse Effect was asymmetrical: Runs of the more conspicuous stimulus substantially depressed performance on subsequent probes of the less conspicuous type; runs of more cryptic positives had much less impact. Similarly asymmetrical effects of a change in caution have been predicted by other authors (GUILFORD \& DAWKINS 1987).

This asymmetry was a prominent feature of the experimental results. An Inverse Effect was evident only following a wheat run; bean runs had little impact on subsequent responses to wheat (Fig. 3). It is possible, therefore, to attribute the Inverse Effect of wheat runs to a change in caution, especially given the additional evidence of effects on error-response times. Although there is clearly an increase in discriminability for the stimulus in the run, our experiment provides no unequivocal evidence for a comparable decrease for stimuli of the opposite type.

\section{Ecological Correlates of Persistence}

In its initial design, this study followed PIETREWICZ \& KAMIL's (1979) protocol, using Treatment Blocks that were randomized mixtures of positive and negative trials. Unlike PIETREWICZ \& KAMIL's (1979) blue jays, however, pigeons did not display an enhanced discrimination with this design. The reason appeared to be that negative trials disrupted any pre-existing attentional state in pigeons. When we substituted a treatment sequence consisting of a contiguous block of positives, the effect emerged immediately. Pigeons may simply have a shorter "attention span" than blue jays in that persistence in attending to a single stimulus may be much reduced. This was also sug- 
gested in the free-response study (BOND 1983), which found that a gap of as little as $150 \mathrm{~ms}$. could be sufficient to cause switching of attentional focus.

If this difference between species is real, it may be of adaptive significance. In ecological terms, pigeons live in a "fine-grained" habitat (Levins 1968): The energetic benefit of any given food item is small and the expectation is for small distances between successive items. In consequence, if a bird is attending to grains of wheat and does not find the next one within a short span of time, it is better off switching its attention to search for some other food.

Blue jays, in contrast, live in a "coarse-grained" habitat: when a jay has found one moth, the next may be a considerable distance away. The energy yield of any given item is larger than for pigeons, but the expectation is that they will not be found close together. In consequence, the value of attentional persistence would be higher for jays, and the birds would be expected to exhibit a longer switching threshold. Confirmation of this hypothesis awaits additional comparative work with fully comparable stimuli, but it suggests that the attention-threshold model (BOND 1983), which is concerned with quantifying attentional persistence, may provide a useful perspective on the adaptive significance of differences between species in visual search and detection.

\section{Acknowledgments}

Our thanks to M. BRown for assistance in protocol development and subject training, S. RANGANATHAN for help in conducting the experiments, and C. LANGLEY for critical editorial advice. This research was supported by Grant No. BNS 84-18740 from the National Science Foundation and Grant No. 1R01 MH44746 from the United States National Institutes of Mental Health.

\section{Appendix A: The Accumulator Model of Visual Search}

\section{Algorithm}

The accumulator model of visual search operates on a series of discrete discrimination trials. The stimulus at each trial is either a negative, $\mathrm{N}$, or a positive, $\mathrm{P}$, and positives are of two types, either A or B. The task of the model is to decide whether a given stimulus is a positive or a negative. Stimuli are discriminated on the basis of serial samples of a variable termed the "stimulus value", $V$. We assumed that $V$ will be approximately normally distributed (Fig. 1a).

The mechanism consists of a pair of accumulators, $\mathrm{AC}_{\mathrm{P}}$ and $\mathrm{AC}_{\mathrm{N}}$, and a corresponding pair of thresholds, $\mathrm{K}_{\mathrm{P}}$ and $\mathrm{K}_{\mathrm{N}}$. The program obtains random samples of $V$, based on its underlying distribution, evaluates them as evidence of the type of stimulus being presented, and adds the evidence into the appropriate accumulator. When the level of accumulated evidence in $\mathrm{AC}_{\mathrm{P}}$ exceeds $\mathrm{K}_{\mathrm{P}}$, sampling ceases, and the model responds "Positive." Likewise, when the level of evidence in $\mathrm{AC}_{\mathrm{N}}$ exceeds $\mathrm{K}_{\mathrm{N}}$, the model responds "Negative." The response time is a function of the number of samples that were required to make the choice.

Most of the elements in any stimulus display in a visual-search experiment are components of the background, and these elements will be the same, whether the 
stimulus is a positive or a negative. What makes the stimulus a positive is the presence, somewhere in the display, of a target item, and the target occupies only a fraction of the display. To simulate this aspect of the task, we added a parameter, $S$, that describes the probability that the sample observation will be drawn from the portion of the display that actually contains the target element.

When the trial is a negative, all samples come from the negative distribution, which is approximately normal, with mean $\mu_{\mathrm{N}}$ and standard deviation $\sigma_{\mathrm{N}}$. When it is a positive, a proportion $S$ of the samples are drawn from a probability distribution characteristic of the given positive type. In the case of a positive of type A, for example, this characteristic distribution has parameters $\mu_{\mathrm{A}}$ and $\sigma_{\mathrm{A}}$. The remaining $1-S$ of the samples are drawn from the negative distribution, with parameters $\mu_{\mathrm{N}}$ and $\sigma_{\mathrm{N}}$. We refer to $S$ as the "salience" of the positive stimulus (Fig. 1b).

An additional problem results from the fact that a searching image task requires detecting targets of several different types, with mean stimulus values that may be scattered above and below the mean value of the background (Fig. 1c). The variance of the characteristic distributions of stimulus values is also much smaller in these studies for the targets than for the background. Moths are more uniform in appearance than tree bark; grains of wheat are more similar to one another than pieces of gravel.

Under these circumstances, a simple arithmetic difference between stimulus values, such as was used in VICKERS' (1979) models, cannot provide a reasonable measure of the likelihood of having drawn a particular sample value from one or another of the stimulus distributions. Instead, the evidentiary variable, $Q$, that is added into the accumulators in our model is derived from the probability, $E$, of the given sample, or one even more deviant, having been drawn from each of the three distributions. In effect, the model determines whether the sample observation is more likely to have come from the $\mathrm{A}$, the $\mathrm{B}$, or the $\mathrm{N}$ distribution. If it is more likely to have come from $\mathrm{A}$ or $\mathrm{B}$, the evidence, $Q$, is added to $\mathrm{AC}_{\mathrm{P}}$; otherwise, it is added to $\mathrm{AC}_{\mathrm{N}}$.

Thus, if the stimulus value obtained in a given sample is $x$, the deviation from $\mu_{\mathrm{N}}$ is $D=\left|\mu_{N}-x\right|$, and the model computes $E_{\mathrm{N}}$ as the probability that $V \leq\left(\mu_{N}-D\right)$ or $V \geq\left(\mu_{N}+D\right)$, given a distribution $\left(\mu_{\mathrm{N}}, \sigma_{\mathrm{N}}\right)$. The same computation is performed for each of the positive distributions, obtaining $E_{\mathrm{A}}$, using $\left(\mu_{\mathrm{A}}, \sigma_{\mathrm{A}}\right)$, and $E_{\mathrm{B}}$, using $\left(\mu_{\mathrm{B}}, \sigma_{\mathrm{B}}\right)$. The model than calculates the difference between the largest of the $E$ values from the positive distributions and the $E$ value obtained from the negative: $Q=E_{N}-\max \left(E_{A}, E_{B}\right)$. If $Q>0$, then $x$ is more likely to have been drawn from the $\mathrm{N}$ distribution than from either the $\mathrm{A}$ or the $\mathrm{B}$ distribution. In this case, a value of $Q$ is added to accumulator $\mathrm{AC}_{\mathrm{N}}$. If $\mathrm{Q}<0$, the evidence is more consistent with $x$ having been drawn from one of the positive distributions. In this case, the absolute value of $Q$ is added to accumulator $\mathrm{AC}_{\mathrm{P}}$.

\section{Methods}

In this model, response bias is a function of the ratio of $K_{P}$ to $K_{N}$. When $K_{P}$ is decreased relative to $K_{N}$, the model requires proportionately less evidence before making a "Positive" response. Given the same underlying stimulus distributions, it will become biased to responding "Positive," and $\boldsymbol{\beta}$, the signal detection criterion, will in- 
crease. Conversely, when $K_{P}$ is increased relative to $K_{N}$, the model will be biased to responding "Negative," and $\boldsymbol{\beta}$ will decrease.

Caution is a function of a weighted sum of $K_{P}$ and $K_{N}$. When either $K_{P}$ or $K_{N}$ is increased, the model will require more evidence before making its decision, and response time will increase. If accuracy was originally limited by a low value of $K_{P}$ and $\mathrm{K}_{\mathrm{N}}$, a shift in caution may also increase accuracy. Conversely, a decrease in either $\mathrm{K}_{\mathrm{P}}$ or $\mathrm{K}_{\mathrm{N}}$ will decrease response time and may also decrease accuracy. Caution and response bias can be manipulated independently by carefully controlling the sum and ratio of the accumulator thresholds.

Changes in discriminability were simulated by varying the salience, which is analogous to increasing the detectability of a visual target by increasing the animal's receptive field. An increase in $S_{\mathrm{A}}$, for example, will increase the proportion of the sample observations from positive stimuli of type A that will have originated from the target item in the stimulus display. As a consequence, evidence of a positive target will accumulate more rapidly for A stimuli, resulting in faster and more accurate responses. The opposite result is produced by decreasing salience: a lower $S_{A}$ will result in a slower accumulation of evidence in the positive accumulator with A stimuli, producing slower and less accurate responses.

In all simulations, half of the stimuli were positives, and the two positive stimulus types were equally abundant. The positive types differed slightly in crypticity, with the more cryptic type being designated as type A and the more conspicuous as type B (Fig. 1b). Several different configurations of relative crypticity were used: $\mu_{\mathrm{A}}$ was set to -0.8 , while $\mu_{\mathrm{B}}$ was varied between 1.5 and 0.85 . $\mu_{\mathrm{N}}$ was always set to $0, \sigma_{\mathrm{N}}$ was set to 1.0 , and $\sigma_{\mathrm{A}}$ and $\sigma_{\mathrm{B}}$ were set to 0.1 . Logarithmic, rather than normal, distributions were used to increase the speed of computation. Except when they were subject to experimental manipulation, $S_{A}$ and $S_{B}$, were set at 0.1 . All simulations used sample sizes of 1000 .

To provide a basis for subsequent manipulations, we first obtained optimal values for $K_{P}$ and $K_{N}$ for each of the stimulus configurations, using nonlinear least squares. The optimization procedure assumed only that responses were unbiased (i.e. that they exhibited a $\boldsymbol{\beta}$ of 1.0) and that similar proportionate changes in speed and accuracy were weighted equally. Experimental trials were subsequently conducted, varying $\mathrm{K}_{\mathrm{P}}$ and $\mathrm{K}_{\mathrm{N}}$ independently by up to $20 \%$ from their optimal levels in each direction to simulate changes in response bias and caution.

Caution was also manipulated independently of response bias by constraining the $\mathrm{K}_{\mathrm{P}} / \mathrm{K}_{\mathrm{N}}$ ratio. The effects of changes in discriminability were tested by systematically varying $S$ for each of the two positive stimulus types between 0.05 and 0.2 . We tested the effects of an increase in $S$ for one of the two positive stimuli alone, as well as in combination with a decrease in $S$ for positives of the opposite type (Inverse Effect). To evaluate the possibility of simultaneous changes in both discriminability and caution, we performed an additional set of optimization trials in which $S$ for one of the two stimuli had been increased to 0.2 .

\section{Literature Cited}

BLough, P. M. 1989: Attentional priming and visual search in pigeons. J. Exp. Psych. Anim. Behav. Proc. 15, 211-223. 
BOND, A. B. 1982: The bead game: Response strategies in free assortment. Human Factors 24, 101-110.

- 1983: Visual search and selection of natural stimuli in the pigeon: The attention threshold hypothesis. J. Exp. Psych. Anim. Behav. Proc. 9, 292-306.

Brown, M. F., COOK, R. G., LAMB, M. R. \& RILEY, D. A. 1984: The relation between response and attentional shifts in pigeon compound matching-to-sample performance. Anim. Learn. Behav. 12, 41-49.

Croze, J. H. 1970: Searching Image in Carrion Crows. Paul Parey, Berlin.

CURIO, E. 1976: The Ethology of Predation. Paul Parey, Berlin.

DAWKINS, M. 1971a: Perceptual changes in chicks: Another look at the "search image" concept. Anim. Behav. 19, 566-574.

— - 1971b: Shifts of "attention" in chicks during feeding. Anim. Behav. 19, 575-582.

FulLICK, T. G. \& GREENWOOD, J. J. D. 1979: Frequency-dependent food selection in relation to two models. Am. Nat. 113, 762-765.

GENDRON, R. P. 1986: Searching for cryptic prey: Evidence for optimal search rates and the formation of search images in quail. Anim. Betrav. 34, 898-912.

— — \& STADDON, J. E. R. 1983: Searching for cryptic prey: The effect of search rate. Am. Nat. 121, 172-186.

— — \& - 1984: A laboratory simulation of foraging behavior: The effect of search rate on the probability of detecting prey. Am. Nat. 124, 407-415.

GetTy, T., KAMIL, A. C. \& REAL, P. G. 1987: Signal detection theory and foraging for cryptic or mimetic prey. In: Foraging Behavior. (KAMIL, A. C., KREBS, J. R. \& Pulliam, H. R., eds.) Plenum Press, New York, pp. 525-548.

Green, D. M. \& Swets, J. A. 1966: Signal Detection Theory and Psychophysics. Krieger, Huntington.

Greenwood, J. J. D. \& Elton, R. A. 1979: Analysing experiments on frequencydependent selection by predators. J. Anim. Ecol. 48, 721-737.

Grizzle, J. E., Starmer, C. F. \& KoCH, G. G. 1969: Analysis of categorical data by linear models. Biometrics 25, 489-504.

GUILFORD, T. \& DAWKINS, M. S. 1987: Search images not proven: A reappraisal of recent evidence. Anim. Bchay. 35, 1838-1845.

HARVEY, P. H., BIRLEY, N. \& BLACKSTOCK, T. H. 1975: The effect of experience on the selective behaviour of song thrushes feeding on artificial populations of $\mathrm{Ce}$ paea (Held). Genetica 45, 211-216.

Kahneman, D. 1973: Attention and Effort. Prentice-Hall, Englewood Cliffs.

KREBS, J. R. 1973: Behavioral aspects of predation. In: Perspectives in Ethology. (BAteson, P. P. G. \& Klopfer, P. H., eds.) Plenum Press, New York, pp. 73111.

LAMB, M. R. 1983: Selective attention in pigeons: effects of cueing on the processing of different types of compound stimuli. Doct. Diss., Univ. of California, Berkeley. Diss. Abs. Int. 44, 345B.

LAWRENCE, E. S. 1985a: Evidence for search image in blackbirds (Turdus merula L.): Short-term learning. Anim. Behav. 33, 929-937.

— - 1985b: Evidence for search image in blackbirds (Turdus merula L.): Long-term learning. Anim. Behav. 33, 1301-1309.

LEVINS, R. 1968: Evolution in Changing Environments: Some Theoretical Explora- 
tions. Princeton Univ. Press, Princeton.

MACKINTOSH, N. J. 1975: A theory of attention: Variations in the associability of stimuli with reinforcement. Psych. Rev. 82, 276-298.

Murdoch, W. W. \& OAten, A. 1974: Predation and population stability. Adv. Ecol. Res. 9, 1-131.

PIETREWICZ, A. T. \& KAMIL, A.C. 1977: Visual detection of cryptic prey by blue jays (Cyanocitta cristata). Science 195, 580-582.

- - 1979: Search image formation in the blue jay (Cyanocitta cristata). Science 204, 1332-1333.

- - 1981: Search images and the detection of cryptic prey: An operant approach. In: Foraging Behavior. (KAMIL, A. C. \& SARgENT, T. D., eds.) Garland STPM Press, New York, pp. 311-332.

RabitTt, P. M. A., CuMming, G. \& VyAs, S. 1977: An analysis of visual search: Entropy and sequential effects. In: Attention and Performance VI. (DORNIC, S., ed.). Lawrence Erlbaum, Hillsdale, pp. 363-386.

RILEY, D. A. \& LEITH, C. R. 1976: Multidimensional psychophysics and selective attention in animals. Psych. Bull. 83, 138-160.

— — \& RoitBlat, H. L. 1978: Selective attention and related cognitive processes in pigeons. In: Cognitive Processes in Animal Behavior. (HulSE, S. H., FowLER, H. \& HoNIG, W. K., eds.) Lawrence Erlbaum, Hillsdale, pp. 249-276.

SAS 1985: SAS User's Guide: Statistics, Version 5 ed. SAS Inst., Cary, SC.

SMITH, P. L. \& VICKERS, D. 1988: The accumulator model of two-choice discrimination. J. Math. Psych. 32, 135-168.

Sutherland, N. S. \& MACKINTOSH, N. J. 1971: Mechanisms of Animal Discrimination Learning. Acad. Press, New York.

TINBERGEN, L. 1960: The natural control of insects in pine woods I. Factors influencing the intensity of predation by songbirds. Arch. Néerland. Zool. 13, 265-343.

VICKERS, D. 1970: Evidence for an accumulator model of psychophysical discrimination. Ergonomics 13, 37-58.

— 1979: Decision Processes in Visual Perception. Acad. Press, New York.

Willis, A. J., Mcewan, J. W. T., Greenwood, J. J. D. \& Elton, R. A. 1980: Food selection by chicks: Effects of colour, density, and frequency of food types. Anim. Behav. 28, 874-879.

Received: July 7, 1990

Accepted: November 5, 1990 (G. Barlow) 